\title{
Use of Vagus Nerve Stimulation and Vagal Maneuvers as Adjuvant Therapy for COVID-19 Patients
}

\author{
Susana Mabel Sendin ${ }^{1}$, Juan Sebastian Yakisich ${ }^{2 *}$ \\ ${ }^{1}$ Outpatient COVID-19 Module, Municipality of Vicente López, Buenos Aires, Argentina. \\ ${ }^{2}$ Department of Pharmaceutical Sciences, School of Pharmacy, Hampton University. Hampton VA (23693), Virginia, United States.
}

Received 17 December 2020; Revised 03 February 2021; Accepted 02 March 2021; Published 27 April 2021

\begin{abstract}
At present there is no effective specific antiviral drug to treat the ongoing COVID-19 pandemic that has already infected millions of individual and caused hundreds of thousand deaths worldwide. There is strong indication that a cytokine storm is responsible for the severity of COVID-19 patients. Pilot studies using IL-6 receptor inhibitors such as Tolicizumab have shown promising results. However, since the cytokine storm is a complex systemic inflammatory response involving multiple cytokines it can be hypothesized that a "paninhibition" of cytokines and/or cytokine receptors will be more effective. However, at the same time this strategy may cause more adverse effects. In this article, we propose the application of Vagus Nerve Stimulation (NVS) and/or some forms of vagal maneuvers as adjuvant therapies to prevent and/or mitigate the cytokine response in COVID-19 patients. This proposal is based on the ability of NVS and to decrease the production of IL-6 and other cytokines. The potential application of the diving response (one form of vagal maneuver), that has been shown to confer intrinsic resistance to inflammation in the blood of diving mammals, is also discussed as adjuvant therapy for COVID-19 patients.
\end{abstract}

Keywords: COVID-19; Cytokines; Cytokine Storm; Vagus Nerve Stimulation; Vagal Maneuvers; Diving Reflex.

\section{Introduction}

The recent COVID-19 pandemic that originated in December 2019 in Wuhan, China has infected 74,378,599 individuals and caused 1,652,235 deaths (https://coronavirus.jhu.edu/map.html, data retrieved on 12/17/2020). At present there are few vaccines approved in US for the prevention [1] but the data on the efficacy and safety in older people is limited [2,3]. In addition, it will take time to distribute the vaccine to the entire population due to limitations of vaccine supplies and trained health personnel [4]. Beside these vaccines, there is not specific treatment for patients with COVID-19 infection [5]. In addition, there is increasing clinical evidence that patients die from complications at present not clearly understood. Beside the expected research on antiviral drugs and vaccines, several drugs are being used in pilot clinical trials in a desperate race to find ways to prevent and mitigate fatal conditions associated with COVID-19 infection. Due to the high number of cases and deaths, simple but effective measures that can be easily applied to decrease the mortality can save thousands of lives. As an example, early application of prone positioning that demonstrated a significant decrease of 28-day and 90-day mortality [6] in patients with severe ARDS has been implemented to treat COVID-19 in several countries such as China [7], Spain [8]. Unfortunately, prone position can be applied to few COVID-19 patients: while the prevalence of ARDS among COVID-19 patients has been reported to be

* Corresponding author: juan.yakisich@hamptonu.edu; yakisich@gmail.com

http://dx.doi.org/10.28991/SciMedJ-2021-03-SI-2

$>$ This is an open access article under the CC-BY license (https://creativecommons.org/licenses/by/4.0/).

(C) Authors retain all copyrights. 
up to $17 \%$ [9], for several reasons, only a fraction of these ADRS patients are actually put in prone positioning [6]. For COVID-19 patients these numbers are similar: prone position was performed in 22 patients (49\% of mechanically ventilated patients) in the Spanish study [8].

It is clear that effective adjuvant therapies that can be applied to a larger fraction of COVID-19 patients will have a greater impact on the current pandemic. In this line, the recent proposal to use melatonin as adjuvant therapy [10], if proven effective, may have a great impact because it could be administered to a large fraction of patients.

Recent findings have implicated the vagus nerve in several aspects of the disease [11-14] and prompted a reevaluation of this important autonomic nerve as potential target to mitigate the severity of the disease. Pharmacological neuromodulation of the vagus nerve to treat COVID-19 patients is an emerging area of research [15], in particular targeting the cholinergic anti-inflammatory pathway, has been proposed in several recent articles [16-19]. In this article we review the rationale behind the use of vagus nerve stimulation (VNS) and/or several forms of vagal maneuvers as adjuvant therapy for COVID-19 patients.

\section{Rationale and Experimental Support for the use of VNS "Early Adjuvant Therapy in COVID-19 Patients"}

There is increasing evidence suggesting that in severe COVID-19 patients, there are mild to severe cytokine storms [20]. Since interleukin-6 is a key player in cytokine storms the use of Tocilizumab (an IL-6 receptor blocker) has been added in several treatment regimes. Results from a single center experience (15 patients) indicate that Tocilizumab appears to be an effective treatment option in COVID-19 patients at risk of cytokine storm [21]. Several case-reports described the successful treatment of COVID-19 with tocilizumab [22-27]. In another study (32 patients) 2 of 5 survivors received Tocilizumab or Sarilumab, another monoclonal antibody against IL-6 [28].

The cytokine storm is a systemic inflammatory response that, in addition of IL-6 other cytokines are involved [29]. Indeed, the severity of COVID-19 depends on the increase in pro-inflammatory factors such as IL-1, IL-2, IL-6, IL-7, IL-10, granulocyte-colony stimulating factor, interferon- $\gamma$-inducible protein 10 , monocyte chemoattractant protein 1 , macrophage inflammatory protein-1 alpha, and TNF- $\alpha$ [5]. Thus, blocking the biological activities of these cytokines, and not only IL-6, may be more beneficial for COVID-19 patients. To achieve this goal a cocktail of cytokine and/or cytokine receptor inhibitors are needed that will likely result in higher incidence of adverse effects. As example, one study reported hypertriglyceridemia as adverse effects in COVID-19 patients receiving Tocilizumab [30].

This is why the ability of VNS to decrease the production of several cytokines can be exploited as a "paninhibitor" of cytokine production in COVID-19 patients.

The "inflammatory reflex," is a well characterized reflex neural circuit mechanism that regulate innate and adaptive immunity by signals traveling the vagus nerve to inhibit monocyte and macrophage production of tumor necrosis factor (TNF) and other cytokines [31]. Electrical stimulation of the vagus nerve has been extensively used in animal models and has FDA approval for the treatment of epilepsy and depression. Recently, Koopman et al. reported that electrical VNS inhibited peripheral blood production of TNF, IL-1 $\beta$, and IL-6 in epilepsy patients. It also significantly inhibited TNF production for up to $84 \mathrm{~d}$ in RA patients [31]. TNF- $\alpha$ was found to be a key mediator of virus-induced $\mathbf{M}_{2}$ muscarinic receptor dysfunction and airway hyperresponsiveness in influenza-infected guinea pigs [32]. Vagus nervestimulating devices are relatively safe and well tolerated. They have not been associated with immunosuppression or long-term complications. They have been used for decades in patients with refractory epilepsy [33, 34] and have been used more recently in patients with depression [35]. Staas et al. reported the first successful outcome of two COVID-19 patients treated with VNS [36]. This encouraging results were confirmed in an independent case report study with two patients using transcutaneous auricular VNS [37]. At present there are 5 registered clinical trials evaluating the benefit of VNS in hospitalized COVID-19 patient (Table 1).

Table 1. Registered clinical trials using VNS to treat COVID-19 patients

\begin{tabular}{lcc}
\hline \multicolumn{1}{c}{ Intervention/Device } & Country & www.clinicalTrials.gov Identifier \# \\
\hline Transcutaneous Auricular Vagus Nerve Stimulation & Argentina & NCT04379037 \\
Auricular neuromodulation & France & NCT04341415 \\
Vagus nerve stimulation using the gammacore neurostimulation device & Spain & NCT04368156 \\
gammaCore@ Sapphire (non-invasive vagus nerve stimulator) & USA & NCT04382391 \\
Yoga-based breathing support & Italy & NCT04413747 \\
\hline
\end{tabular}

Although VNS may not constitute a cure it has the potential to be used as adjuvant therapy at earlier stages to avoid the progression of the disease to more severe stages. The application at early stages may decrease the number of patients the need to be admitted to ICU, improve outcomes and decrease mortality in ICU patients. In later stages may help to mitigate the severity of the symptoms and sequelae in COVID-19 survivors. 


\section{Rationale and Theoretical Basis for the use of Vagal Maneuvers in COVID-19 Patients}

It is important to point out that the term "Vagus Nerve Stimulation" refers to any technique that stimulates the nerve vagus including: a) Manual massage and compression of the carotid artery in the cervical region of the neck (Carotid sinus massage, CSM) and b) several forms of paced breathing. However, at present the impact of these techniques on cytokine production has not been evaluated. Strictly speaking, CMS is one of the several "Vagal Maneuvers" that increase vagal parasympathetic tone and have several clinical applications. Vagal maneuvers include among others, carotid sinus massage, Valsalva maneuver, diving response and the oculocardiac reflex.

It has been reported that vagal maneuvers induce different degree of cardiac inhibition in humans. For instance, the percent heart rate (beats/min) change produced by the diving response (seated) was -43 and by CSM was only -17 [38]. These data suggest that not all "Vagal maneuvers" will induce similar changes on inflammatory cytokines and that further research is needed to evaluate the impact of specific vagal maneuver on cytokine secretion.

Despite the lack of data on the above mentioned forms of vagal maneuvers on cytokine production, they deserve closer attention for the following reasons: i) CSM was shown to be effective in suppressing seizures [39] and is a widely used method for termination of supraventricular tachycardia (SVT) [40], ii) Cardio-respiratory VNS may explain the positive emotional and cognitive benefits attributed to deep breathing, yoga, or aerobic exercise activities, iii) Stimulation of the vagus nerve by acupuncture in a mouse model of endotoxemia has shown anti-inflammatory effects: The authors reported that manual acupuncture decreased the levels of TNF alpha in serum as well as decreased levels of TNF alpha mRNA and protein in spleen [41].

From the practical point of view, vagal maneuvers can be easily implemented immediately during the current pandemic without major accommodations and economic limitations. Careful consideration should be taken regarding contraindications and adverse effect. In general vagal maneuvers are contraindicated in patients with unstable SVT and there are specific contraindications for particular maneuvers. For instance, CSM is contraindicated in patients with carotid bruits and patients with a history of transient ischemic attack (TIA) or stroke [42]. In these patients other types of vagal maneuvers could be used. The diving response or diving reflex is particularly interesting. The diving response is a protective autonomic response observed in marine mammals and also in humans, as a physiologic change to decrease oxygen expenditure [42,43]. Remarkably, it was observed that the blood of healthy northern elephant seals and Weddell seals (two deep-diving mammals) possess intrinsic anti-inflammatory properties: in these species the production of IL6 elicited by LPS exposure was 50 to 500 times lower compared to healthy human blood [44]. These observations suggest that the diving response may have a dual beneficial effect on COVID-19 patients: on one hand it may make the blood intrinsically resistant to inflammation (perhaps by a decrease in the production of cytokines and therefore mitigating the cytokine storm). On the other hand, it may decrease the oxygen consumption in the cells of COVID-19 patients that are already under relatively hypoxia. In fact, it is believed that some of the effects of the diving response is to limit oxygen consumption by non-essential muscle groups as well as to induce bradychardia to further limit unnecessary oxygen consumption [43]. Indeed the diving response has been shown to have an $\mathrm{O}_{2}$-conserving effect in humans [45]. Niehues and Klovenski [42] provided more detailed and practical information on several vagal maneuvers including techniques.

\section{Rationale for the Potential Benefit of VNS and Vagal Maneuvers at Earlier Stages of the Disease}

At present there are four registered clinical trials aimed to evaluate the potential benefit of electrical VNS in hospitalized COVID-19 patients but not in ambulatory asymptomatic or with mild stages of the disease (Table 1).

VNS and vagal maneuvers may be beneficial at earlier stages in outpatients to prevent or at least partially mitigate the development of the cytokine storm. The rationale for this assumption is supported by the fact that when COVID-19 patients are admitted in the ICU they already have elevated levels of IL- 6 that could have been suppressed by vagal maneuvers at home or in outpatient offices. Indeed, it was recently demonstrated that VNS reduced the blood levels of IL-6 in COVID-19 patients [37]. In addition, patients can be quickly trained to perform some form of less risky and less complicated vagal maneuver such as the diving response.

\section{Conclusion}

The rationale to consider electrical VNS in COVID-19 patients is supported by several studies in animal models of inflammation and by at least by two experimental studies in humans. While there is no experimental evidence that nonelectrical stimulation of the nerve vagus by clinically used vagal maneuvers decrease inflammation or production of cytokines in humans, all of them have the potential to do so because they all share a similar underlying mechanism of action. This proposal suggests the possibility of a pilot clinical trial during the current pandemic to first evaluate the potential use of VNS and vagal maneuvers in COVID-19 patients and later, if successful, to find the best alternative for each particular patient. 


\section{Declarations}

\subsection{Author Contributions}

Conceptualization, S.M.S. and J.S.Y.; formal analysis, S.M.S. and J.S.Y.; writing - review and editing, S.M.S. and J.S.Y. All authors have read and agreed to the published version of the manuscript.

\subsection{Funding}

The authors received no financial support for the research, authorship, and/or publication of this article.

\subsection{Ethical Approval}

The manuscript does not contain experiments on animals and humans; hence ethical permission not required.

\subsection{Data Availability Statement}

Data sharing not applicable: No new data were created or analyzed in this study.

\subsection{Conflict of Interest}

The authors declare that they have no known competing financial interests or personal relationships that could have appeared to influence the work reported in this paper.

\section{References}

[1] Tanne, J. H. (2020) Covid-19: Pfizer-BioNTech vaccine is rolled out in US. Bmj, 371 m4836. doi:10.1136/bmj.m4836.

[2] Cabanillas, B., Akdis, C. and Novak, N. (2020) Allergic reactions to the first COVID-19 vaccine: a potential role of Polyethylene glycol? Allergy. doi:10.1111/all.14711.

[3] Soiza, R. L., Scicluna, C. and Thomson, E. C. (2020) Efficacy and safety of COVID-19 vaccines in older people. Age Ageing. doi:10.1093/ageing/afaa274.

[4] Mills, M. C. and Salisbury, D. (2020) The challenges of distributing COVID-19 vaccinations. EClinicalMedicine 100674. doi:10.1016/j.eclinm.2020.100674.

[5] Zhang, J., Xie, B. and Hashimoto, K. (2020) Current status of potential therapeutic candidates for the COVID-19 crisis. Brain Behav Immun. doi:10.1016/j.bbi.2020.04.046.

[6] Guerin, C., Reignier, J., Richard, J. C., Beuret, P., Gacouin, A., Boulain, T., Mercier, E., Badet, M., Mercat, A., Baudin, O., Clavel, M., Chatellier, D., Jaber, S., Rosselli, S., Mancebo, J., Sirodot, M., Hilbert, G., Bengler, C., Richecoeur, J., Gainnier, M., Bayle, F., Bourdin, G., Leray, V., Girard, R., Baboi, L. and Ayzac, L. (2013) Prone positioning in severe acute respiratory distress syndrome. N Engl J Med, 368(23), 2159-2168. doi:10.1056/NEJMoa1214103.

[7] Meng, L., Qiu, H., Wan, L., Ai, Y., Xue, Z., Guo, Q., Deshpande, R., Zhang, L., Meng, J., Tong, C., Liu, H. and Xiong, L. (2020) Intubation and Ventilation amid the COVID-19 Outbreak: Wuhan's Experience. Anesthesiology. doi:10.1097/aln.0000000000003296.

[8] Barrasa, H., Rello, J., Tejada, S., Martin, A., Balziskueta, G., Vinuesa, C., Fernandez-Miret, B., Villagra, A., Vallejo, A., Sebastian, A. S., Cabanes, S., Iribarren, S., Fonseca, F. and Maynar, J. (2020) SARS-Cov-2 in Spanish Intensive Care: Early Experience with 15-day Survival In Vitoria. Anaesth Crit Care Pain Med. doi:10.1016/j.accpm.2020.04.001.

[9] Chen, N., Zhou, M., Dong, X., Qu, J., Gong, F., Han, Y., Qiu, Y., Wang, J., Liu, Y., Wei, Y., Xia, J., Yu, T., Zhang, X. and Zhang, L. (2020) Epidemiological and clinical characteristics of 99 cases of 2019 novel coronavirus pneumonia in Wuhan, China: a descriptive study. Lancet, 395(10223), 507-513. doi:10.1016/s0140-6736(20)30211-7.

[10] Zhang, R., Wang, X., Ni, L., Di, X., Ma, B., Niu, S., Liu, C. and Reiter, R. J. (2020) COVID-19: Melatonin as a potential adjuvant treatment. Life Sci, 250 117583. doi:10.1016/j.lfs.2020.117583.

[11] Chigr, F., Merzouki, M. and Najimi, M. (2020) Autonomic Brain Centers and Pathophysiology of COVID-19. ACS Chem Neurosci, 11(11), 1520-1522. doi:10.1021/acschemneuro.0c00265.

[12] Dey, J., Alam, M. T., Chandra, S., Gupta, J., Ray, U., Srivastava, A. K. and Tripathi, P. P. (2020) Neuroinvasion of SARS-CoV2 may play a role in the breakdown of respiratory center of the brain. J Med Virol. doi: 10.1002/jmv.26521.

[13] Jakhmola, S., Indari, O., Chatterjee, S. and Jha, H. C. (2020) SARS-CoV-2, an Underestimated Pathogen of the Nervous System. SN Compr Clin Med 1-10. doi:10.1007/s42399-020-00522-7.

[14] Maurier, F., Godbert, B. and Perrin, J. (2020) Respiratory Distress in SARS-CoV-2 without Lung Damage: Phrenic Paralysis Should Be Considered in COVID-19 Infection. Eur J Case Rep Intern Med, 7(6), 001728. doi:10.12890/2020_001728. 
[15] Pavlov, V. A. (2020) The evolving obesity challenge: Targeting the vagus nerve and the inflammatory reflex in the response. Pharmacol Ther 107794. doi:10.1016/j.pharmthera.2020.107794.

[16] Ahmad, F. (2020) COVID-19 induced ARDS, and the use of galantamine to activate the cholinergic anti-inflammatory pathway. Med Hypotheses, 145 110331. doi:10.1016/j.mehy.2020.110331.

[17] Andersson, U. (2020) The cholinergic anti-inflammatory pathway alleviates acute lung injury. Mol Med, $26(1)$, 64. doi:10.1186/s10020-020-00184-0.

[18] Bonaz, B., Sinniger, V. and Pellissier, S. (2020) Targeting the cholinergic anti-inflammatory pathway with vagus nerve stimulation in patients with Covid-19? Bioelectron Med, 6 15. doi:10.1186/s42234-020-00051-7.

[19] Ten Hove, A. S., Brinkman, D. J., Li Yim, A. Y. F., Verseijden, C., Hakvoort, T. B. M., Admiraal, I., Welting, O., van Hamersveld, P. H. P., Sinniger, V., Bonaz, B., Luyer, M. D. and de Jonge, W. J. (2020) The role of nicotinic receptors in SARSCoV-2 receptor ACE2 expression in intestinal epithelia. Bioelectron Med, 6(1), 20. doi:10.1186/s42234-020-00057-1.

[20] Ye, Q., Wang, B. and Mao, J. (2020) The pathogenesis and treatment of the 'Cytokine Storm' in COVID-19. J Infect. doi:10.1016/j.jinf.2020.03.037.

[21] Luo, P., Liu, Y., Qiu, L., Liu, X., Liu, D. and Li, J. (2020) Tocilizumab treatment in COVID-19: A single center experience. J Med Virol. doi:10.1002/jmv.25801.

[22] Cellina, M., Orsi, M., Bombaci, F., Sala, M., Marino, P. and Oliva, G. (2020) Favorable changes of CT findings in a patient with COVID-19 pneumonia after treatment with tocilizumab. Diagn Interv Imaging. doi:10.1016/j.diii.2020.03.010.

[23] De Luna, G., Habibi, A., Deux, J. F., Colard, M., d'Alexandry d'Orengiani, A., Schlemmer, F., Joher, N., Kassasseya, C., Pawlotsky, J. M., Ourghanlian, C., Michel, M., Mekontso-Dessap, A. and Bartolucci, P. (2020) Rapid and Severe Covid-19 Pneumonia with Severe Acute Chest Syndrome in a Sickle Cell Patient Successfully Treated with Tocilizumab. Am J Hematol. doi:10.1002/ajh.25833.

[24] Fontana, F., Alfano, G., Mori, G., Amurri, A., Lorenzo, T., Ballestri, M., Leonelli, M., Facchini, F., Damiano, F., Magistroni, R. and Cappelli, G. (2020) Covid-19 pneumonia in a kidney transplant recipient successfully treated with Tocilizumab and Hydroxychloroquine. Am J Transplant. doi:10.1111/ajt.15935.

[25] Michot, J. M., Albiges, L., Chaput, N., Saada, V., Pommeret, F., Griscelli, F., Balleyguier, C., Besse, B., Marabelle, A., Netzer, F., Merad, M., Robert, C., Barlesi, F., Gachot, B. and Stoclin, A. (2020) Tocilizumab, an anti-IL6 receptor antibody, to treat Covid-19-related respiratory failure: a case report. Ann Oncol. doi: 10.1016/j.annonc.2020.03.300.

[26] Mihai, C., Dobrota, R., Schroder, M., Garaiman, A., Jordan, S., Becker, M. O., Maurer, B. and Distler, O. (2020) COVID-19 in a patient with systemic sclerosis treated with tocilizumab for SSc-ILD. Ann Rheum Dis, 79(5), 668-669. doi:10.1136/annrheumdis-2020-217442.

[27] Zhang, X., Song, K., Tong, F., Fei, M., Guo, H., Lu, Z., Wang, J. and Zheng, C. (2020) First case of COVID-19 in a patient with multiple myeloma successfully treated with tocilizumab. Blood Adv, 4(7), 1307-1310. doi:10.1182/bloodadvances.2020001907.

[28] Jacobs, J. P., Stammers, A. H., St Louis, J., Hayanga, J. W. A., Firstenberg, M. S., Mongero, L. B., Tesdahl, E. A., Rajagopal, K., Cheema, F. H., Coley, T., Badhwar, V., Sestokas, A. K. and Slepian, M. J. (2020) Extracorporeal Membrane Oxygenation in the Treatment of Severe Pulmonary and Cardiac Compromise in COVID-19: Experience with 32 patients. Asaio j. doi:10.1097/mat.0000000000001185.

[29] Behrens, E. M. and Koretzky, G. A. (2017) Review: Cytokine Storm Syndrome: Looking Toward the Precision Medicine Era. Arthritis Rheumatol, 69(6), 1135-1143. doi:10.1002/art.40071.

[30] Morrison, A. R., Johnson, J. M., Ramesh, M., Bradley, P., Jennings, J. and Smith, Z. R. (2020) Letter to the Editor: Acute hypertriglyceridemia in patients with COVID-19 receiving tocilizumab. J Med Virol. doi:10.1002/jmv.25907.

[31] Koopman, F. A., Chavan, S. S., Miljko, S., Grazio, S., Sokolovic, S., Schuurman, P. R., Mehta, A. D., Levine, Y. A., Faltys, M., Zitnik, R., Tracey, K. J. and Tak, P. P. (2016) Vagus nerve stimulation inhibits cytokine production and attenuates disease severity in rheumatoid arthritis. Proc Natl Acad Sci U S A, 113(29), 8284-8289. doi:10.1073/pnas.1605635113.

[32] Nie, Z., Scott, G. D., Weis, P. D., Itakura, A., Fryer, A. D. and Jacoby, D. B. (2011) Role of TNF-alpha in virus-induced airway hyperresponsiveness and neuronal $\mathrm{M}(2)$ muscarinic receptor dysfunction. $\mathrm{Br} \mathrm{J}$ Pharmacol, 164(2b), 444-452. doi:10.1111/j.1476-5381.2011.01393.x.

[33] Flesler, S., Reyes, G., Fortini, S., Ramos, B., Cersósimo, R., Bartuluchi, M., \& Caraballo, R. H. (2017). Estimulador del nervio vago: tratamiento en 158 pacientes pediátricos con un largo seguimiento. Revista de Neurología, 64(11), 496. doi:10.33588/rn.6411.2016458.

[34] Revesz, D., Rydenhag, B. and Ben-Menachem, E. (2016) Complications and safety of vagus nerve stimulation: 25 years of experience at a single center. J Neurosurg Pediatr, 18(1), 97-104. doi:10.3171/2016.1.Peds15534. 
[35] Bottomley, J. M., LeReun, C., Diamantopoulos, A., Mitchell, S. and Gaynes, B. N. (2019) Vagus nerve stimulation (VNS) therapy in patients with treatment resistant depression: A systematic review and meta-analysis. Compr Psychiatry, 98152156. doi:10.1016/j.comppsych.2019.152156.

[36] Staats, P., Giannakopoulos, G., Blake, J., Liebler, E. and Levy, R. M. (2020) Use of non-invasive vagus nerve stimulation to treat respiratory symptoms associated with COVID-19: A theoretical hypothesis and early clinical experience. Neuromodulation. doi:10.1111/ner.13172.

[37] Boezaart, A. P. and Botha, D. A. (2020) Treatment of Stage 3 COVID-19 With Transcutaneous Auricular Vagus Nerve Stimulation Drastically Reduces Interleukin-6 Blood Levels: A Report on Two Cases. Neuromodulation. doi:10.1111/ner.13293.

[38] Arnold, R. W. (1999). The human heart rate response profiles to five vagal maneuvers. The Yale journal of biology and medicine, 72(4), 237-244.

[39] Laine Green, A. and Weaver, D. F. (2014) Vagal stimulation by manual carotid sinus massage to acutely suppress seizures. J Clin Neurosci, 21(1), 179-180. doi:10.1016/j.jocn.2013.03.017.

[40] Collins, N. A. and Higgins, G. L., 3rd. (2015) Reconsidering the effectiveness and safety of carotid sinus massage as a therapeutic intervention in patients with supraventricular tachycardia. Am J Emerg Med, 33(6), 807-809. doi:10.1016/j.ajem.2015.02.047.

[41] Lim, H. D., Kim, M. H., Lee, C. Y. and Namgung, U. (2016) Anti-Inflammatory Effects of Acupuncture Stimulation via the Vagus Nerve. PLoS One, 11(3), e0151882. doi:10.1371/journal.pone.0151882.

[42] Niehues, L. J. and Klovenski, V. (2020), StatPearls. StatPearls Publishing, StatPearls Publishing LLC., Treasure Island (FL).

[43] Godek, D. and Freeman, A. M. (2020), StatPearls. StatPearls Publishing. StatPearls Publishing LLC., Treasure Island (FL).

[44] Bagchi, A., Batten, A. J., Levin, M., Allen, K. N., Fitzgerald, M. L., Huckstadt, L. A., Costa, D. P., Buys, E. S. and Hindle, A. G. (2018) Intrinsic anti-inflammatory properties in the serum of two species of deep-diving seal. J Exp Biol, 221(Pt 13). doi:10.1242/jeb.178491.

[45] Alboni, P., Alboni, M. and Gianfranchi, L. (2011) Diving bradycardia: a mechanism of defence against hypoxic damage. J Cardiovasc Med (Hagerstown), 12(6), 422-427. doi:10.2459/JCM.0b013e328344bcdc. 